\title{
MASS CULTURE OF MOSQUITOLARVAE (CULEX PIPIENS)AND THEIR NUTRITIVE VALUE AS FOOD FOR THE FREHSWATER PRAWN
}

\author{
Madlen M. Habashy ${ }^{\mathrm{J}}$ and Suzan Daba ${ }^{2}$ \\ 1- National Institute of Oceanography and Fisheries, Fish Research Station,Qanater El- \\ Khayriya, Egypt. \\ 2 - Research and Training Center on Vectors of Diseases, Ain Shams \\ University, Egypt.
}

Key words: Culture, mosquito larvae, natural food, freshwater prawn, nutritive value.

\begin{abstract}
T arvae of Culex pipiens, mosquito were cultured under controlled Llaboratory conditions. They were introduced to larvae of the freshwater prawn, Macrobrachium rosenbergii (De Man) in a 7 5-day feeding experiment. Larvae of the prawn with an initial total length of 1.2 $\mathrm{cm}$ and initial body weight of $0.02 \mathrm{~g}$, received three feeding regimes; (1) live Artemia nauplii alone, Dl (2) live mosquito larvae alone,D2 (3) mixture of Artemia nauplii and mosquito larvae (1:1) (D3). Each treatment was evaluated in duplicate aquaria. The growth rate, survival and amino acids analysis were performed for prawns larvae fed on different diets. No significant difference $(\mathrm{P}>0.05)$ was found in growth rates, survival, and amino acid composition of prawns larvae receiving DlandD3.

A significantly lower growth and survival rates were observed in prawns larvae fed on mosquito larvae as a sole food and this was due to cannibalism that occurred in this group. The nutritive value and proximate composition of mosquito larvae were measured (crude protein, $42.2 \%$ and crude lipid, $16.1 \%$ and high values of most essential amino acids content such as threonine 5.08, valine 5.20, histidine 2.83, isoleucine 6.96, phenylalanine 4.46 and lysine $7.59 \%$ ).Also, mosquito larvae contained some major metals such as $\mathrm{Ca}, \mathrm{Na}, \mathrm{K}, \mathrm{Mg}, \mathrm{Fe}, \mathrm{Cu}, \mathrm{Zn}$ and $\mathrm{Mn}$. The study revealed that mosquito larvae in their live form failed to improve the growth rate and survival of the freshwater prawn when added to Artemia nauplii. This may be due to their active swimming and breathing behavior.
\end{abstract}

